PAPER

Improved constraints on violations of the Einstein equivalence principle in the electromagnetic sector with complementary cosmic probes

To cite this article: R F L Holanda et al 2017 Class. Quantum Grav. 34195003

View the article online for updates and enhancements.
Related content

A test for cosmic distance duality R.F.L. Holanda, R.S. Gonçalves and J.S. Alcaniz

Probing the distance-duality relation with high- $z$ data

R.F.L. Holanda, V.C. Busti, F.S. Lima et al.

X-rav surface brightness observations of galaxy clusters, cosmic opacity and the limits on the matter density parameter R.F.L. Holanda, Kamilla V.R.A. Silva and V.C. Busti

Recent citations

- Galaxy clusters and a possible variation of the fine structure constant

L.R. Colaço et al

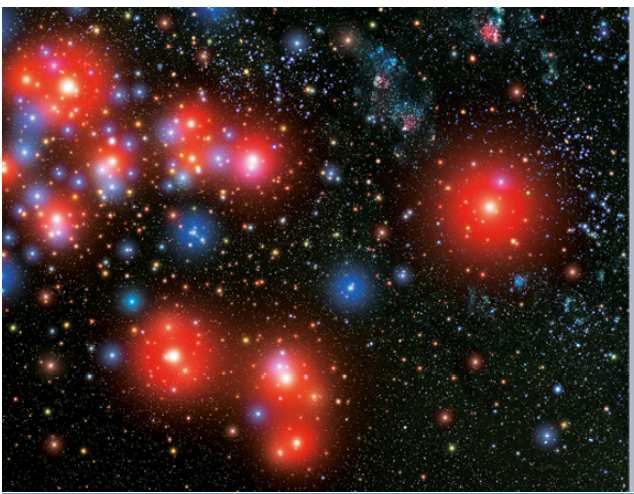

$\frac{\mathbf{A} \mid \mathbf{A}}{\mathbf{S}} \mid$ IOP Astronomy ebooks

Part of your publishing universe and your first choice for astronomy, astrophysics, solar physics and planetary science ebooks. iopscience.org/books/aas 


\title{
Improved constraints on violations of the Einstein equivalence principle in the electromagnetic sector with complementary cosmic probes
}

\author{
R F L Holanda ${ }^{1,2,3}$, S H Pereira ${ }^{4}$, V C Busti ${ }^{5,6}$ \\ and $\mathrm{CH} \mathrm{G} \mathrm{Bessa}{ }^{7}$ \\ ${ }^{1}$ Departamento de Física, Universidade Federal de Sergipe, 49100-000, \\ Aracaju—SE, Brazil \\ 2 Departamento de Física, Universidade Federal de Campina Grande, 58429-900, \\ Campina Grande-PB, Brazil \\ ${ }^{3}$ Departamento de Física, Universidade Federal do Rio Grande do Norte, 59300-000, \\ Natal-RN, Brazil \\ ${ }^{4}$ Departamento de Física e Química, Universidade Estadual Paulista (Unesp), \\ Faculdade de Engenharia, Guaratinguetá, Av. Dr. Ariberto Pereira da Cunha 333, \\ 12516-410—Guaratinguetá, SP, Brazil \\ 5 Department of Physics and Astronomy, University of Pennsylvania, Philadelphia, \\ PA 19104, United States of America \\ ${ }^{6}$ Departamento de Física Matemática, Universidade de São Paulo, Rua do Matão \\ 1371, São Paulo-SP, 05508-090, Brazil \\ ${ }^{7}$ Departamento de Física, Universidade Federal da Paraíba, 58051-970, João \\ Pessoa-PB, Brazil \\ E-mail: holandarfl@gmail.com, shpereira@feg.unesp.br, busti@sas.upenn.edu \\ and carlos@ cosmos.phy.tufts.edu
}

Received 9 May 2017, revised 18 August 2017

Accepted for publication 24 August 2017

Published 11 September 2017

\section{Abstract}

Recent results have shown that a field non-minimally coupled to the electromagnetic Lagrangian can induce a violation of the Einstein equivalence principle. This kind of coupling is present in a very wide class of gravitation theories. In a cosmological context, this would break the validity of the cosmic distance duality relation as well as cause a time variation of the fine structure constant. Here, we improve constraints on this scenario by using four different observables: the luminosity distance of type Ia supernovae, the angular diameter distance of galaxy clusters, the gas mass fraction of galaxy clusters and the temperature of the cosmic microwave background at different redshifts. We consider four standard parametrizations adopted in the literature and show that, due to a high complementarity of the data, the errors are shrunk 
between $20 \%$ and $40 \%$ depending on the parametrization. We also show that our constraints are weakly affected by the geometry considered to describe the galaxy clusters. In short, no violation of the Einstein equivalence principle is detected up to redshifts $\sim 3$.

Keywords: cosmology, general relativity theory modified, distance scale

(Some figures may appear in colour only in the online journal)

\section{Introduction}

Modified gravity theories have appeared recently as an alternative to General Relativity (GR) when the last one faces some difficulties to explain some observations, as the accelerated expansion of the Universe, galactic velocities in galaxy clusters or rotational curves of spiral galaxies. Among such new theories we can cite massive gravity theories [1], modified Newtonian dynamic (MOND) [2], $f(R, T)$ theories [3], models with extra dimensions, as brane world models, Kaluza-Klein theories, string and loop quantum cosmology theories [4-6]. Nevertheless, some of these new theories naturally break the Einstein equivalence principle (EEP), leading to observational consequences that deserve to be tested and verified.

Hees et al [7-9] have shown that a class of theories that explicitly breaks the EEP can be tested using recent observational data. Particularly, those theories motivated by scalar-tensor theories of gravity, which introduce an additional coupling between the Lagrangian of the usual non-gravitational matter field with a new scalar field [10-24]. In this class of theories, all the electromagnetic sector is affected, leading to a variation in the value of the fine structure constant ( $\alpha=\alpha_{0} \zeta(z)$, where $\alpha_{0}$ is the current value) of the quantum electrodynamics [25, 26], a non-conservation of the photon number and, consequently, a modification of the expression of the luminosity distance, $D_{L}(z)$, important for various cosmological estimates. In this context, the so-called cosmic distance duality relation, $D_{L}(1+z)^{-2} D_{A}^{-1}=1=\eta$, where $D_{A}$ is the angular diameter distance, as well as the Cosmic Microwave Background (CMB) radiation temperature evolution law, $T_{\mathrm{CMB}}(z)=T_{0}(1+z)$, are also affected. These variations are intimately and unequivocally linked (see next section).

Based on the results of [7-9], some recent papers [27-29] have also searched, using observational data, for signatures of that class of modified gravity theories which explicitly breaks the EEP. The authors have used angular diameter distances (ADD) of galaxy clusters obtained via their X-ray surface brightness jointly with observations of the Sunyaev-Zel'dovich effect (SZE) [30, 31], SNe Ia samples [32], CMB temperature in different redshifts, $T_{\mathrm{CMB}}(z)[33,34]$ and the most recent $x$-ray gas mass fraction (GMF) data with galaxy clusters in the redshift range $0.078 \leqslant z \leqslant 1.063$ [35]. In the [27] it was considered ADD + SNe Ia sample, in [28] it was used $\mathrm{ADD}+\mathrm{SNe} \mathrm{Ia}+T_{\mathrm{CMB}}$ and in the [28], GMF $+\mathrm{SNe} \mathrm{Ia}+T_{\mathrm{CMB}}$. The crucial point in these papers is that the dependence of the SZE/X-ray technique and GMF measurements on possible departure from $\eta=\zeta=1$ was taken into account (see section 3 for details). The main result found was that no significant deviation for the EEP was verified by means of the electromagnetic sector, although the results do not completely rule out those models. Thus, additional tests are still required.

In this paper, we continue searching for deviations of the EEP by considering several cosmological observations and the class of models that explicitly breaks the EEP in the electromagnetic sector discussed in [7-9] . We consider a more complete analysis, including $\mathrm{ADD}+\mathrm{GMF}+\mathrm{SNe} \mathrm{Ia}+T_{\mathrm{CMB}}$ data. However, in our analyses the GMF measurements are 
used by using two methods: in the method I we use GMF measurements obtained separately via X-ray and SZE observations for a same galaxy cluster and in the method II the X-ray GMF observations of galaxy clusters are used jointly with SNe Ia data. Therefore, it is important to emphasize that we not only combine previous tests but add one more: the method I. As result, this more comprehensive analysis due to a larger data set allowed us to decrease the errors roughly by $20 \%$ to $40 \%$ depending on the adopted functional form for the deviation. Once more we show that no significant deviation of the EEP is verified.

This paper is organized as follows: section 2 we briefly revise the cosmological equations for a class of scalar-tensor theories based on [8]. The consequences for cosmological measurements are presented in section 3 . The cosmological data are in section 4 , and the analises and results in section 5. We finish with a conclusion in section 6 .

\section{Scalar-tensor theories coupled to electromagnetic sector}

A specific class of modified gravity theories characterized by a universal non-minimal coupling between an extra scalar field $\Phi$ to gravity was studied recently by Hees et al [7-9]. In such scalar-tensor theories the standard matter Lagrangian $\mathcal{L}_{i}$ and the scalar field $\Phi$ are represented by the action:

$$
S=\int \mathrm{d}^{4} x \sqrt{-g}\left[f_{i}(\Phi) \mathcal{L}_{i}\left(g_{\mu \nu}, \Psi_{i}\right)+\frac{1}{2 \kappa}\left(\Phi R-\frac{\omega(\Phi)}{\Phi}\left(\partial_{\sigma} \Phi\right)^{2}-V(\Phi)\right)\right],
$$

where $R$ is the Ricci scalar for the metric $g_{\mu \nu}$ with determinant $g, \kappa=8 \pi G$, where $G$ is the gravitational constant, $V(\Phi)$ is the scalar-field potential, $f_{i}(\Phi)$ and $\omega(\Phi)$ are arbitrary functions of $\Phi . \mathcal{L}_{i}$ is the matter Lagrangian for the non-gravitational fields $\Psi_{i}$, where for a matter content consisting of a perfect fluid, for instance, we have $\mathcal{L}_{\text {Mat }}\left(g_{\mu \nu}, \Psi_{\text {Mat }}\right)$, where $\Psi_{\text {Mat }}$ stands for the field describing the perfect fluid. For the electromagnetic radiation we have $\mathcal{L}_{\mathrm{EM}}\left(g_{\mu \nu}, \Psi_{\mathrm{EM}}\right)$, where $\Psi_{\mathrm{EM}}=A^{\mu}$ stands for the 4-vector potential. From the extremization of the action (1) follows the Einstein field equations [8]:

$R_{\mu \nu}-\frac{1}{2} g_{\mu \nu} R=\kappa \frac{f_{i}(\Phi)}{\Phi} T_{\mu \nu}^{i}+\frac{1}{\Phi}\left[\nabla_{\mu} \nabla_{\nu}-g_{\mu \nu} \square\right] \Phi+\frac{\omega(\Phi)}{\Phi^{2}}\left[\partial_{\mu} \Phi \partial_{\nu} \Phi-\frac{1}{2} g_{\mu \nu}\left(\partial_{\alpha} \Phi\right)^{2}\right]-g_{\mu \nu} \frac{V(\Phi)}{2 \Phi}$,

where the stress-energy tensor is given by $T_{\mu \nu}^{i}=(-2 / \sqrt{-g}) \delta\left(\sqrt{-g} \mathcal{L}_{i}\right) / \delta g^{\mu \nu}$. It is clear that the cases $f_{i}(\Phi) \neq 1$ and/or $\Phi \neq 1$ will represent the break of EEP, while the limit $\Phi \rightarrow 1$, $f_{i}(\Phi) \rightarrow 1, \omega(\Phi)=0$ and $V(\Phi)=0$ corresponds to the standard framework, for some matter Lagrangian. The case $\omega(\Phi)=$ constant and $f_{i}(\Phi)=1$ stands for the Brans-Dicke theory [24]. The dilaton [10] and pressuron theory [36] also follows from that action.

In order to study just the break of EEP due to a coupling of a single scalar field $\Phi$ with the electromagnetic sector of theory, which is motivated by the first term of the action (1) or the first term on the right side of (2), we just need to consider the usual electromagnetic Lagrangian coupled to $\Phi$ through $f_{i}(\Phi)$. In which follows we will present the main results in the case where the electromagnetic field is the only matter field present into the action (1), although it is not a significant source of curvature (photons are just test particles non-minimally coupled to $\Phi)$. In the vacuum the Lagrangian is [9]

$$
\mathcal{L}_{\mathrm{EM}}\left(g_{\mu \nu}, A^{\mu}\right)=-\frac{1}{4} F^{\mu \nu} F_{\mu \nu},
$$


with $F^{\mu \nu}=\partial^{\mu} A^{\nu}-\partial^{\nu} A^{\mu}$ and we will consider a coupling $f_{i}(\Phi)=f_{\mathrm{EM}}(\Phi)$. Variation of the action with respect to the 4-potential $A^{\mu}$ gives the modified Maxwell equations

$$
\nabla_{\nu}\left(f_{\mathrm{EM}}(\Phi) F^{\mu \nu}\right)=0 .
$$

Following the standard procedure in GR [37, 38], we expand the 4-potential as $A^{\mu}=\Re\left\{\left(b^{\mu}+\epsilon c^{\mu}+O\left(\epsilon^{2}\right)\right) \exp ^{i \theta / \epsilon}\right\}$ and use the Lorenz gauge which leads to the usual null-geodesic at leading order. The next order of the modified Maxwell equations is given by

$$
\begin{aligned}
& k^{\nu} \nabla_{\nu} b=-\frac{1}{2} b \nabla_{\nu} k^{\nu}-\frac{1}{2} b k^{\nu} \partial_{\nu} \ln f_{\mathrm{EM}}(\Phi) \\
& k^{\nu} \nabla_{\nu} h^{\mu}=\frac{1}{2} k^{\mu} h^{\nu} \partial_{\nu} \ln f_{\mathrm{EM}}(\Phi)
\end{aligned}
$$

where $b$ is the amplitude of $b^{\mu}=b h^{\mu}, h^{\mu}$ is the polarisation vector and $k_{\mu} \equiv \partial_{\mu} \theta$. The conservation law of the number of photons is written as

$$
\nabla_{\nu}\left(b^{2} k^{\nu}\right)=-b^{2} k^{\nu} \partial_{\nu} \ln f_{\mathrm{EM}}(\Phi) .
$$

The wave vector in the flat FRW metric in spherical coordinate is $k^{\mu}=\left(k^{0}, k^{r}, 0,0\right)=\left(-k_{0}, k_{0} / a(t), 0,0\right)$ and it can be showed that the quantity $K=b(t, r) r a(t) \sqrt{f_{\mathrm{EM}}(\Phi(t))}$ is constant along a geodesic.

The flux of energy comes from the $T^{0 i}$ component of the energy momentum tensor and is given by

$$
F_{0}=\left|a_{0} b^{2} k^{0} k^{r}\right|=\frac{k_{r}^{2} b^{2}}{a_{0}^{2}}=\frac{k_{r}^{2} K^{2}}{r_{0}^{2} a_{0}^{4} f_{\mathrm{EM}}\left(\Phi_{0}\right)}=\frac{C}{r_{0}^{2} a_{0}^{4} f_{\mathrm{EM}}\left(\Phi_{0}\right)}
$$

where $C$ is a constant. The emitted flux is

$$
F_{e}=\frac{C}{r_{e}^{2} a_{e}^{4} f_{\mathrm{EM}}\left(\Phi_{e}\right)}
$$

where the index $e$ refers to the emitted signal. The angular integral of this defines the luminosity $L_{e}$

$$
L_{e}=\frac{4 \pi C}{a_{e}^{2} f_{\mathrm{EM}}\left(\Phi_{e}\right)} .
$$

Finally, the expression for the distance of luminosity is

$$
D_{L}=\left(\frac{L_{e}}{4 \pi F_{0}}\right)^{1 / 2}=\frac{a_{0}}{a_{e}} a_{0} r_{0} \sqrt{\frac{f_{\mathrm{EM}}\left(\Phi_{0}\right)}{f_{\mathrm{EM}}\left(\Phi_{e}\right)}}=c(1+z) \sqrt{\frac{f_{\mathrm{EM}}\left(\Phi_{0}\right)}{f_{\mathrm{EM}}(\Phi(z))}} \int_{0}^{z} \frac{\mathrm{d} z}{H(z)} .
$$

Such expression clearly shows that $D_{L}$ is slightly modified for a non-minimal coupling $f_{\mathrm{EM}}$ between the electromagnetic Lagrangian and an extra scalar field.

On the other hand, the angular diameter distance $D_{A}$ is a purely geometric quantity that is the same as in ordinary electromagnetism

$$
D_{A}(z)=\frac{c}{(1+z)} \int_{0}^{z} \frac{\mathrm{d} z^{\prime}}{H\left(z^{\prime}\right)} .
$$


By comparing with (11) we have:

$$
\frac{D_{L}(z)}{D_{A}(z)(1+z)^{2}}=\sqrt{\frac{f_{\mathrm{EM}}\left(\Phi_{0}\right)}{f_{\mathrm{EM}}(\Phi(z))}} \equiv \eta(z),
$$

where we have defined the parameter $\eta(z)$ related to $f_{\mathrm{EM}}(\Phi(z))$ for convenience, when $\eta(z)=1$, the above relation is also known as the cosmic distance duality relation (CDDR). The CDDR is a relation between angular diameter and luminosity distances for a given redshift, $z$, namely, $D_{L} D_{A}^{-1}(1+z)^{-2}=1$. This equation is an astronomical consequence deduced from the reciprocity theorem when photons follow null geodesics, the geodesic deviation equation is valid and the number of photons is conserved. It plays an essential role in cosmological observations and in the last years it has been tested by several authors in different cosmological context [39-44] (see table I in [45] for recent results).

As commented earlier, the kind of coupling explored in this paper leads to a variation in the value of the fine structure constant, $\alpha=\alpha_{0} \zeta(z)$, violations of the cosmic distance duality relation, $D_{L}(1+z)^{-2} D_{A}^{-1}=\eta(z)$, as well as a modification in the CMB temperature evolution law, $T_{\mathrm{CMB}}(z)=T_{0}(1+z)^{1-\tau}$, and these possible variations are intimately and unequivocally linked. As shown in [7] (see their equations (12) and (34)), if one parametrizes a possible departure from the CDDR validity with a $\eta(z)$ term, the consequent deviation in the CMB temperature evolution law and the temporal evolution of the fine structure constant have to be described by

$$
\frac{\Delta \alpha}{\alpha}=\zeta(z)-1=\eta^{2}(z)-1
$$

and

$$
T(z)=T_{0}(1+z)\left[0.88+0.12 \eta^{2}(z)\right] .
$$

Deviation from such relations will have several consequences on cosmological observables, as modifications of angular diameter distances of galaxy clusters obtained via their x-ray and SZE observations, the gas mass fraction of galaxy clusters and the CMB temperature evolution law. Thus we have a robust method to test the break of the equivalence principle in the electromagnetic sector.

In this work, in order to better explore possible break of EEP, we consider four widely used parametrizations for the $\eta(z)$ function:

- P1: $\eta(z)=1+\eta_{0} z$

- P2: $\eta(z)=1+\eta_{0} z /(1+z)$

- P3: $\eta(z)=(1+z)^{\eta_{0}}$

- P4: $\eta(z)=1+\eta_{0} \ln (1+z)$

where $\eta_{0}$ is the parameter to be constrained and the limit $\eta_{0}=0$ (or $\left.\eta(z)=1\right)$ corresponds to no violation of the EEP.

\section{Consequences for cosmological measurements}

In the following sections, we discuss the consequences of the EEP breaking on cosmological measurements and explain the basic equations used in our analyses. 


\subsection{Angular diameter distance of galaxy clusters}

The angular diameter distance of galaxy clusters can be obtained from their SunyaevZel' dovich effect (SZE) [46] and x-ray observations [31, 47]. In this point, it is important to discuss the key points of this technique. The SZE is a small distortion of the CMB spectrum, due to the inverse Compton scattering of the $\mathrm{CMB}$ photons passing through a population of hot electrons in intra-cluster medium. The temperature decrement in Rayleigh-Jeans portion of $\mathrm{CMB}$ radiation spectrum that crosses the center of the cluster is given by ${ }^{8}$

$$
\Delta T_{0} \equiv T_{0} f\left(\nu, T_{\mathrm{e}}\right) \frac{\sigma_{\mathrm{T}} k_{\mathrm{B}} T_{\mathrm{e}}}{m_{\mathrm{e}} c^{2}} n_{e 0} \sqrt{\pi} \theta_{c} D_{A} g(\beta / 2),
$$

with

$$
g(\alpha) \equiv \frac{\Gamma[3 \alpha-1 / 2]}{\Gamma[3 \alpha]},
$$

where $\Gamma(\alpha)$ is the gamma function, $\theta_{c}$ is the cluster core angular size, $n_{e 0}$ is the central electronic density of the intra-cluster medium, $k_{\mathrm{B}}$ the Boltzmann constant, $T_{e}$ is the electronic temperature, $T_{0}=2.728 \mathrm{~K}$ is the present-day temperature of the $\mathrm{CMB}, f\left(\nu, T_{e}\right)$ accounts for frequency shift and relativistic corrections [49], and $m_{e}$ the electron mass. The Thompson cross section, $\sigma_{\mathrm{T}}$, can be written in terms of the fine structure constant $\left(\alpha=e^{2} / c \hbar\right)$ as [50]

$$
\sigma_{\mathrm{T}}=\frac{8 \pi \hbar^{2}}{3 m_{e}^{2} c^{2}} \alpha^{2}
$$

where $e$ is the electronic charge, $c$ is the speed of light and $\hbar$ is the reduced Planck constant.

On the other hand, the x-ray emission is due to thermal bremsstrahlung and the central surface brightness is given by [51]

$$
S_{X 0} \equiv \frac{D_{A}^{2} \Lambda_{e}}{D_{L}^{2} 4 \sqrt{\pi}} n_{e 0}^{2} \theta_{c} D_{A} g(\beta),
$$

which clearly depends on the CDDR [51]. The term $\Lambda_{e}$ is the central x-ray cooling function of the intra-cluster medium [52]. Thus, the angular diameter distance of a galaxy cluster can be found by eliminating $n_{e 0}$ in the equations (16) and (19), taking the validity of the CDDR and considering the constancy of $\alpha$. However, if one considers $\alpha=\alpha_{0} \zeta(z)$ and $D_{L} D_{A}^{-1}(1+z)^{-2}=\eta(z) \neq 1$, a more general result appears [51, 53]

$D_{A}(z)=\left[\frac{\Delta T_{0}^{2}}{S_{\mathrm{X} 0}}\left(\frac{m_{\mathrm{e}} c^{2}}{k_{\mathrm{B}} T_{e}}\right)^{2} \frac{g(\beta)}{g(\beta / 2)^{2} \theta_{\mathrm{c}}}\right] \times\left[\frac{\Lambda_{e} \zeta^{3}(z)}{4 \pi^{3 / 2} f\left(\nu, T_{\mathrm{e}}\right)^{2}\left(T_{0}\right)^{2} \sigma_{\mathrm{T}}^{2}(1+z)^{4}} \frac{1}{\zeta^{4}(z) \eta(z)^{2}}\right]$.

The observational quantity in the above equation is

$D_{A}^{\text {data }}(z)=\left[\frac{\Delta T_{0}^{2}}{S_{\mathrm{X} 0}}\left(\frac{m_{\mathrm{e}} c^{2}}{k_{\mathrm{B}} T_{e}}\right)^{2} \frac{g(\beta)}{g(\beta / 2)^{2} \theta_{\mathrm{c}}}\right] \times\left[\frac{\Lambda_{e}}{4 \pi^{3 / 2} f\left(\nu, T_{\mathrm{e}}\right)^{2}\left(T_{0}\right)^{2} \sigma_{\mathrm{T}}^{2}(1+z)^{4}}\right]$.

So, the currently measured quantity is $D_{A}^{\text {data }}(z)=D_{A}(z) \eta^{2}(z) \zeta(z)$, where $D_{A}(z)$ is the true angular diameter distance. In this way, by considering the large class of theories proposed by $[7,9]$ where the relation (14) is valid, we have access to [27]

\footnotetext{
${ }^{8}$ For simplicity, we assume the spherical $\beta$-model to the galaxy clusters [48], where the electron density of the hot intra-cluster gas has a profile of the form: $n_{e}(r)=n_{e 0}\left[1+\left(\frac{r}{r_{c}}\right)^{2}\right]^{-3 \beta / 2}$.
} 


$$
\frac{D_{L}}{(1+z)^{2} D_{A}^{\text {data }}(z)}=\eta^{-3}(z) .
$$

By using the equation above, we define the distance modulus of a galaxy cluster (GC) data as

$$
\mu_{G C}(\eta, z)=5 \log \left[\eta^{-3}(z) D_{A}^{\text {data }}(z)(1+z)^{2}\right]+25 .
$$

Thus, if we have SNe Ia distance modulus measurements, $\mu(z)$, at identical redshifts of galaxy clusters, we can put observational constraints on the $\eta$ parameter.

\subsection{Gas mass fraction of galaxy clusters}

Here, we can put limits on $\eta(z)$ from two methods:

3.2.1. Method I. The gas mass fraction is defined as [54]

$$
f_{\text {gas }}=\frac{M_{\text {gas }}}{M_{\text {tot }}},
$$

where $M_{\text {tot }}$ is the total mass and $M_{\text {gas }}$ is the gas mass obtained by integrating the gas density model, for instance, the spherical $\beta$-model. Under the hydrostatic equilibrium, isothermality and the spherical $\beta$-model assumptions, the $M_{\text {tot }}$ within a $R$ radius is given by [55]

$$
M_{\mathrm{tot}}(<R)=\frac{3 \beta k_{\mathrm{B}} T_{e}}{\mu_{m} G m_{H}}\left[\frac{R^{3}}{\left(r_{c}^{2}+R^{2}\right)}\right],
$$

where $\mu_{m}$ and $m_{H}$ are, respectively, the total mean molecular weight and the proton mass, $G$ is the gravitational constant and $r_{c}$ is the cluster core radius. On the other hand, the $M_{\text {gas }}$ within a volume $V$ is obtained by

$$
M_{\mathrm{gas}}(<V)=\frac{8 \pi n_{e 0} m_{H} r_{c}^{3}}{(1+X)} I_{M}(y, \beta),
$$

where

$$
I_{M}\left(R / r_{c}, \beta\right) \equiv \int_{0}^{R / r_{c}}\left(1+x^{2}\right)^{-\frac{3 \beta}{2}} x^{2} \mathrm{~d} x,
$$

$x=r / r_{c}$ and $X$ is the hydrogen abundance. In this way, the gas mass fraction of a galaxy cluster is

$$
f_{\text {gas }}=\frac{8 \pi m_{H}^{2} \mu_{m} G n_{e 0}}{3(1+X) \beta k_{\mathrm{B}} T_{G}}\left[\frac{\left(r_{c}^{5}+r_{c}^{3} R^{2}\right)}{R^{3}}\right] I_{M}\left(\frac{R}{r_{c}}, \beta\right) .
$$

The quantity $n_{e 0}$ in the above equation can be determined from two different kinds of observations: x-rays surface brightness and the Sunyaev-Zeldovich effect.

By Using SZE observations, the central electron density can be expressed as [56]

$$
n_{e 0}^{\mathrm{SZE}}=\frac{\Delta T_{0} m_{e} c^{2} \Gamma\left(\frac{3}{2} \beta\right)}{f_{\left(\nu, T_{e}\right)} T_{\mathrm{CMB}} \sigma_{\mathrm{T}} k_{\mathrm{B}} T_{e} D_{\mathrm{A}} \pi^{1 / 2} \Gamma\left(\frac{3}{2} \beta-\frac{1}{2}\right) \theta_{c}} .
$$

From the equation (18), one may show that the current gas mass fraction measurements via SZE depend on $\alpha$ as [57] 


$$
f_{\mathrm{SZE}}^{\mathrm{obs}} \propto \alpha^{-2},
$$

or still, if $\alpha=\alpha_{0} \zeta(z)$,

$$
f_{\mathrm{SZE}}^{\mathrm{th}} \propto[\zeta(z)]^{-2} .
$$

On the other hand, from X-ray observations, the bolometric luminosity is given by [52]

$$
L_{x}=\left(\frac{2 \pi k_{\mathrm{B}} T_{e}}{3 m_{e}}\right)^{\frac{1}{2}} \frac{2^{4} e^{6}}{3 \hbar m_{e} c^{3}} g_{\mathrm{B}}\left(T_{e}\right) \frac{2}{(1+X)} 4 \pi n_{e 0} \int_{0}^{R}\left(1+\frac{r^{2}}{r_{c}^{2}}\right)^{-3 \beta} r^{2} \mathrm{~d} r .
$$

Defining

$$
I_{L}\left(R / r_{c}, \beta\right) \equiv \int_{0}^{R / r_{c}}\left(1+x^{2}\right)^{-3 \beta} x^{2} \mathrm{~d} x,
$$

we obtain the equation for the bolometric luminosity

$$
L_{x}=\left(\frac{2 \pi k_{\mathrm{B}} T_{e}}{3 m_{e}}\right)^{\frac{1}{2}} \frac{2^{4} e^{6}}{3 \hbar m_{e} c^{3}} g_{\mathrm{B}}\left(T_{e}\right) \frac{2}{(1+X)} 4 \pi n_{e 0}^{2} r_{c}^{3} I_{L}\left(R / r_{c}, \beta\right),
$$

which can be rewritten in terms of $\alpha$ as

$$
L_{x}=\alpha^{3}\left(\frac{2 \pi k_{\mathrm{B}} T_{e}}{3 m_{e}}\right)^{\frac{1}{2}} \frac{2^{4} \hbar^{2}}{3 m_{e}} g_{\mathrm{B}}\left(T_{e}\right) \frac{2}{(1+X)} 4 \pi n_{e 0}^{2} D_{A}^{2} \theta_{c}^{2} r_{c} I_{L}\left(R / r_{c}, \beta\right),
$$

where $D_{A}$ is the angular diameter distance, $n_{e}$ is the electronic density of gas, $g_{\mathrm{B}}$ is the Gaunt factor which takes into account the corrections due quantum and relativistic effects of Bremsstrahlung emission. However, the quantity $L_{x}$, the total x-ray energy per second leaving the galaxy cluster, is not an observable. The quantity observable is the x-ray flux

$$
F^{x}=L_{x} / 4 \pi D_{L}^{2},
$$

where $D_{L}$ is the luminosity distance. Thus, as one may see from equations (35) and (36), $n_{e 0}$ is $\propto \alpha^{-3 / 2} D_{L} / D_{A}$. Therefore, if $\alpha=\alpha_{0} \zeta(z)$ and the cosmic distance duality relation is $D_{L}(1+z)^{-2} / D_{A}=\eta(z)$, the gas mass fraction measurements extracted from x-ray data are affected by a possible departure of $\alpha_{0}$ and $\eta(z)=1$, such as [57, 58]

$$
f_{\mathrm{x}-\text { ray }}^{\text {th }} \propto[\zeta(z)]^{-3 / 2} \eta(z)
$$

As discussed in [57], current $f_{\mathrm{x}-\text { ray }}^{\text {obs }}$ and $f_{\mathrm{SZE}}^{\text {obs }}$ measurements have been obtained by assuming $\zeta(z)=1$ and $\eta(z)=1$. However, if $\alpha$ varies over the cosmic time, the real gas mass fraction from x-ray $\left(f_{\mathrm{x}-\text { ray }}^{\text {th }}\right)$ and SZE $\left(f_{\mathrm{SZE}}^{\text {th }}\right)$ observations should be related with the current observations by

$$
\begin{aligned}
& f_{\mathrm{x}-\text { ray }}^{\text {th }}=\zeta(z)^{-3 / 2} \eta(z) f_{\mathrm{x}-\mathrm{ray}}^{\mathrm{obs}}, \\
& f_{\mathrm{SZE}}^{\mathrm{th}}=\zeta(z)^{-2} f_{\mathrm{SZE}}^{\mathrm{obs}} .
\end{aligned}
$$

In this way, as one would expect, $f_{\text {gas }}$ measurements from both techniques have to agree with each other since they are measuring the very same quantity $\left(f_{\mathrm{x}-\text { ray }}^{\text {th }}=f_{\mathrm{SZE}}^{\text {th }}\right)$. Thus, the expression relating current $\mathrm{X}$-ray and SZE gas mass fraction observations is given by:

$$
f_{\mathrm{SZE}}^{\mathrm{obs}}=\zeta(z)^{1 / 2} \eta(z) f_{\mathrm{x}-\mathrm{ray}}^{\mathrm{obs}} .
$$


Therefore, by using equation (14) [7, 9], we have access to

$$
f_{\mathrm{SZE}}^{\mathrm{obs}}(z)=\eta(z)^{2} f_{\mathrm{x}-\mathrm{ray}}^{\mathrm{obs}} .
$$

If one has $f_{\mathrm{SZE}}^{\mathrm{obs}}$ and $f_{\mathrm{x}-\text { ray }}^{\text {obs }}$ for the same galaxy cluster it is possible to impose limits on $\eta(z)$.

3.2.2. Method II. Since galaxy clusters are the largest virialized objects in the Universe, one may expect that their cluster baryon fraction is a faithful representation of the cosmological average baryon fraction $\Omega_{b} / \Omega_{M}$, in which $\Omega_{b}$ and $\Omega_{M}$ are, respectively, the fractional mass density of baryons and all matter. Thus, x-ray observations of galaxy clusters can be used to constrain cosmological parameters if one assumes that $f_{\text {gas }}$ is the same at all $z$ [54]. For this context, $\mathrm{x}$-ray gas mass fraction observations of galaxy clusters are used to constrain cosmological parameters from an expression [59-61] that depends on the CDDR, such as [62]:

$$
f_{\mathrm{x}-\text { ray }}^{\text {obs }}(z)=N\left[\frac{D_{L}^{*} D_{A}^{* 1 / 2}}{D_{L} D_{A}^{1 / 2}}\right],
$$

where the symbol $*$ denotes the quantities from a fiducial cosmological model (usually a flat $\Lambda \mathrm{CDM}$ model where $\eta=1$ ) that are used in the observations and the normalization factor $N$ carries all the information about the matter content in the cluster. In the [62], the authors showed that this quantity is affected by a possible departure from $\eta=1$ and the equation (42) must be rewritten as

$$
f_{\mathrm{x}-\text { ray }}^{\text {obs }}(z)=N\left[\frac{\eta^{1 / 2}(z) D_{L}^{* 3 / 2}}{D_{L}^{3 / 2}}\right],
$$

where the $\eta(z)$ parameter appears after using $D_{L} D_{A}^{-1}(1+z)^{-2}=\eta(z)$.

However, as discussed earlier, the [57] showed that the gas mass fraction measurements extracted from $\mathrm{x}$-ray data are also affected by a possible departure of $\zeta(z)=1$, such as

$$
f_{\mathrm{x}-\text { ray }}^{\text {th }} \propto[\zeta(z)]^{-3 / 2}
$$

or, by considering the equation (14),

$$
f_{\mathrm{x}-\text { ray }}^{\text {th }} \propto \eta(z)^{-3} \text {. }
$$

In this context, the quantity $f_{\mathrm{x}-\text { ray }}^{\text {obs }}$ may still deviate from its true value by a factor $\eta^{-3}$, which does not have a counterpart on the right side of the equation (43). Then, this expression has to be modified to [29]

$$
f_{\mathrm{x}-\text { ray }}^{\text {obs }}(z)=N\left[\frac{\eta^{7 / 2}(z) D_{L}^{* 3 / 2}}{D_{L}^{3 / 2}}\right] .
$$

Finally, the luminosity distance of a galaxy cluster can be obtained from its gas mass fraction by

$$
D_{L}=\eta^{7 / 3}(z) D_{L}^{*}\left[N / f_{\mathrm{x}-\text { ray }}^{\text {obs }}(z)\right]^{2 / 3},
$$

and so, its distance modulus is

$$
\mu_{G C}(\eta, z)=5 \log \left[\eta^{7 / 3}(z) D_{L} *\left[N / f_{\mathrm{x}-\text { ray }}^{\text {obs }}(z)\right]^{2 / 3}\right]+25
$$

Again, if we have SNe Ia distance module measurements, $\mu(z)$, at identical redshifts of galaxy clusters, we can put observational constraints on the $\eta(z)$ parameter. 

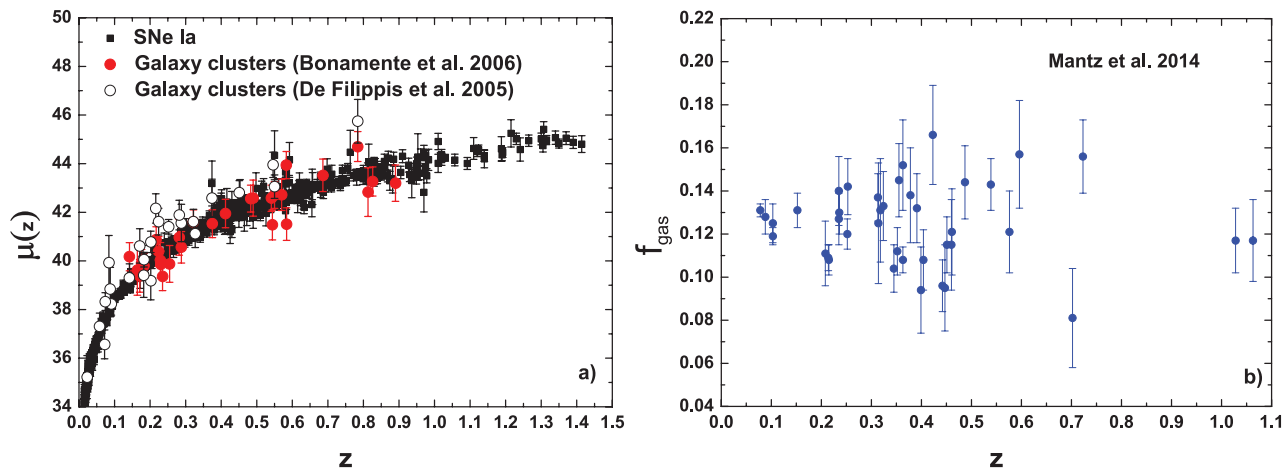

Figure 1. In figure (a) we plot the 580 distance moduli (black squares) of SNe Ia [32], 29 (red circle) and 25 (open circle) distance moduli of galaxy clusters from [30, 31], respectively. In figure (b) we plot the 40 gas mass fraction (GMF) data [35].

\subsection{CMB temperature evolution law}

The last and more simple modified equation is the CMB temperature evolution law $T_{\mathrm{CMB}}(z)$. According to the $[7,9]$, the standard CMB temperature evolution law, $T_{\mathrm{CMB}}(z)=T_{0}(1+z)$, has been modified to

$$
T_{\mathrm{CMB}}(z)=T_{0}(1+z)\left[1+0.12 \eta(z)^{2}\right],
$$

if one considers violations of the cosmic distance duality relation such as $D_{L}(1+z)^{-2} D_{A}^{-1}=$ $\eta(z)$.

\section{Cosmological data}

In our analysis, we use the following data set:

- 29 x-ray and SZE gas mass fraction measurements as given in [56] (see figure 2(a)). Actually, the sample consists of 38 massive galaxy clusters spanning redshifts from 0.14 up to 0.89 . In order to perform a realistic model for the cluster gas distribution, the gas density was modeled with the non-isothermal double $\beta$-model that generalizes the single $\beta$-model profile. An important aspect concerning the galaxy cluster sample shown in figure $1(\mathrm{c})$ is that some objects are not well described by the hydrostatic equilibrium model (see table 6 in [31]). They are: Abell 665, ZW 3146, RX J1347.5-1145, MS 1358.4 + 6245, Abell 1835, MACS J1423 + 2404, Abell 1914, Abell 2163, Abell 2204. By excluding these objects from our sample, we end up with a subsample of 29 galaxy clusters. Moreover, it is worth mentioning that the shape parameters of the gas density model $\left(\theta_{c}\right.$ and $\beta$ ) were obtained from a joint analysis of the X-ray and SZE data, which makes the SZE gas mass fraction not independent ${ }^{9}$. However, simulations have shown that the values of $\theta_{c}$ and $\beta$ computed separately by SZE and x-ray observations agree at $1 \sigma$ level within a radius $r_{2500}$, the same used in the La Roque et al [56] observations.

- Two samples of angular diameter distance of galaxy clusters obtained via their $\mathrm{SZE}+\mathrm{x}$-ray observations. These samples are different from each other by the

\footnotetext{
${ }^{9}$ In all data with SZE observations used in our analysis the frequency used to obtain the SZE signal in galaxy clusters sample considered was $30 \mathrm{GHz}$, in this band the effect on the SZE from a variation of $T_{\mathrm{CMB}}$ is completely negligible [65]. Therefore, we do not consider a modified CMB temperature evolution law in the galaxy cluster data.
} 

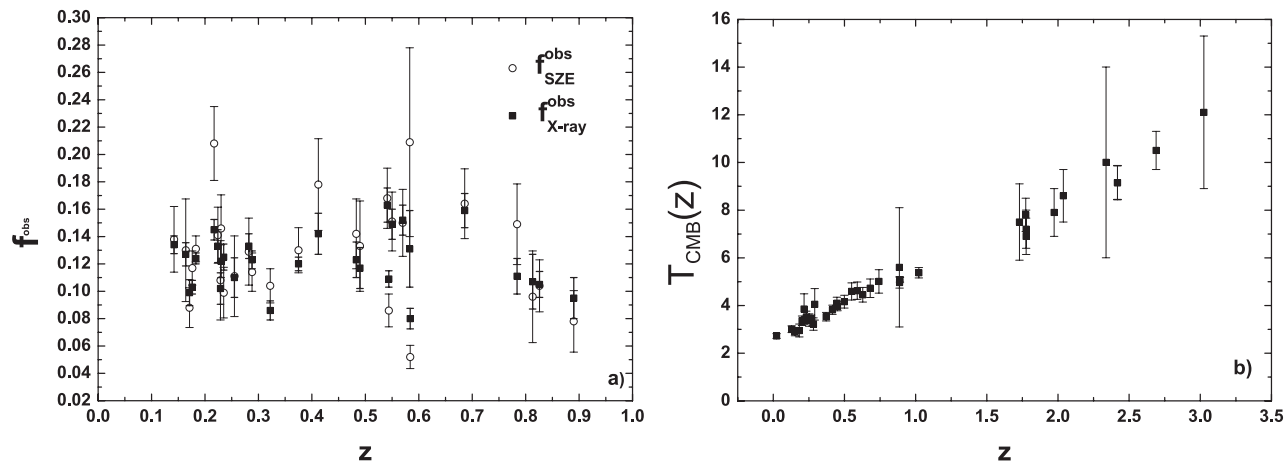

Figure 2. In figure (a) we plot the 29 GMF from [56]. In figure (b) we plot the 36 $T_{\mathrm{CMB}}(z)$ data $[33,34]$.

assumptions used to describe the clusters (see figure 1(a)). The first one corresponds to 29 angular diameter distances of galaxy clusters compiled by [31]. The 29 galaxy clusters here are identical to those in the previous item, where the gas density was also modeled with the non-isothermal double $\beta$-model. The second one is that presented by the [30], where the $\mathrm{x}$-ray surface brightness was described by an elliptical isothermal $\beta$-model. In this case, the galaxy clusters are distributed over the redshift interval $0.023 \leqslant z \leqslant 0.784$. It is critical to consider different assumptions on the galaxy clusters morphology since the distance depends on the hypotheses considered. In both samples, we have added a conservative $12 \%$ of systematic error (see table 3 in [31]).

- The most recent x-ray mass fraction measurements of 40 galaxy clusters in redshift range $0.078 \leqslant z \leqslant 1.063$ from the [35] (see figure 1(b)). These authors measured the gas mass fraction in spherical shells at radii near $r_{2500}{ }^{10}$, rather than integrated at all radii $\left(<r_{2500}\right)$ as in previous works. The effect of this is to significantly reduce the corresponding theoretical uncertainty in the gas depletion from hydrodynamic simulations (see figure 6 in their paper and also $[63,64])$.

- The Union2.1 compilation SNe Ia sample [32] formed by $580 \mathrm{SNe}$ Ia data in the redshift range $0.015 \leqslant z \leqslant 1.4$ (see figure 1(a)), fitted using SALT2 [66]. All analysis and cuts were developed in a blind manner, i.e. with the cosmology hidden. In this point it is important to detail our methodology: we need SNe Ia and galaxy clusters at identical redshifts. Thus, for each galaxy cluster, we select SNe Ia with redshifts obeying the criterion $\left|z_{G C}-z_{S N e}\right| \leqslant 0.005$ and calculate the following weighted average for the SNe Ia data:

$$
\bar{\mu}=\frac{\sum\left(\mu_{i} / \sigma_{\mu_{i}}^{2}\right)}{\sum 1 / \sigma_{\mu_{i}}^{2}}, \sigma_{\bar{\mu}}^{2}=\frac{1}{\sum 1 / \sigma_{\mu_{i}}^{2}} .
$$

Then, we end up with 40, 29 and $25 \bar{\mu}_{i}$ and $\sigma_{\bar{\mu}_{i}}^{2}$ measurements when the sample from [35, 31] and [30] is considered, respectively. Following [32] we added quadratically a 0.15 systematic error to each $\mathrm{SNe}$ Ia distance modulus error.

- The $T_{\mathrm{CMB}}(z)$ sample is composed by 36 points (see figure $2(\mathrm{~b})$ ). The data at low redshifts are from SZE observations [33] and at high redshifts from observations of spectral lines [34]. In total, this represents 36 observations of the CMB temperature at redshifts between 0 and 2.5. We also use the estimation of the current $\mathrm{CMB}$ temperature $T_{0}=2.725 \pm 0.002$ $\mathrm{K}$ [67] from the CMB spectrum as estimated from the COBE satellite.

${ }^{10}$ This radius is the one within which the mean cluster density is 2500 times the critical density of the Universe at the cluster's redshift. 

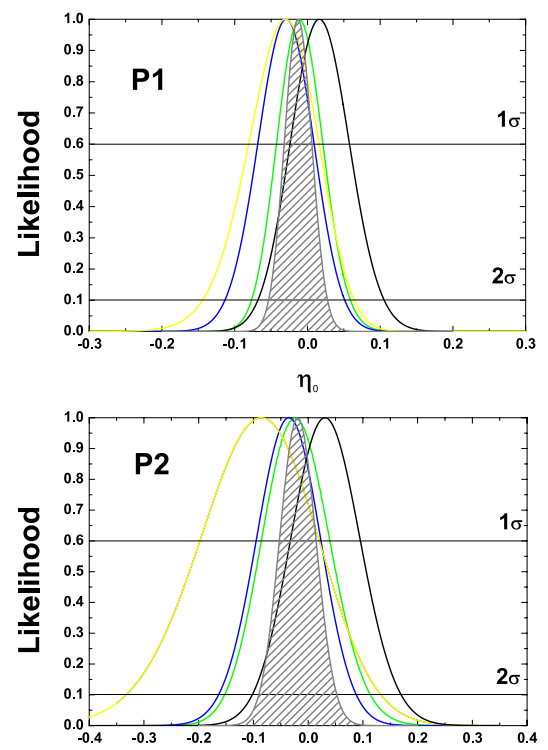

$\eta_{0}$

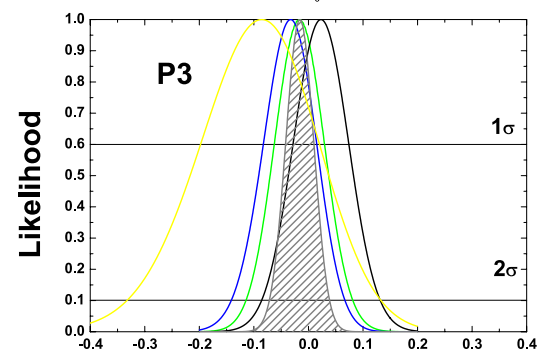

$\eta_{0}$

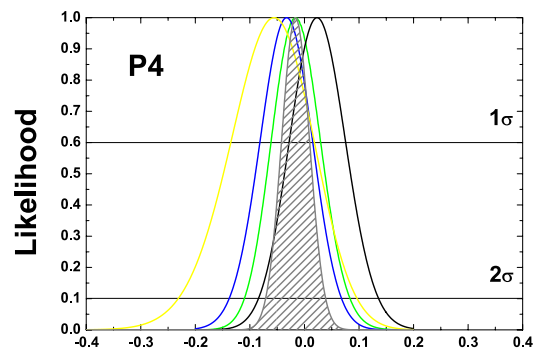

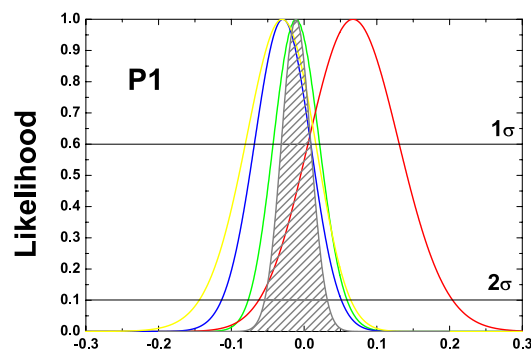

$\eta_{0}$

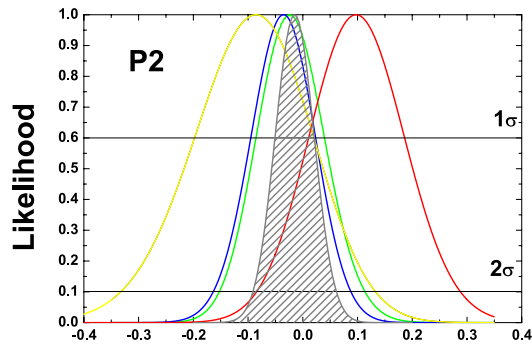

$\eta_{0}$

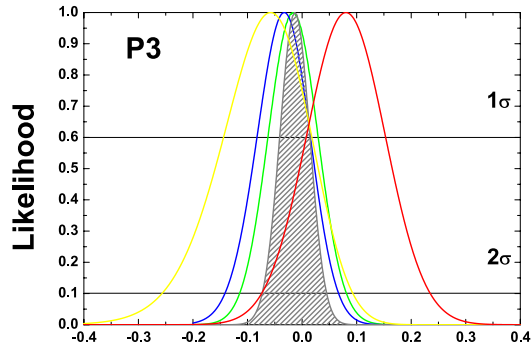

$\eta_{0}$

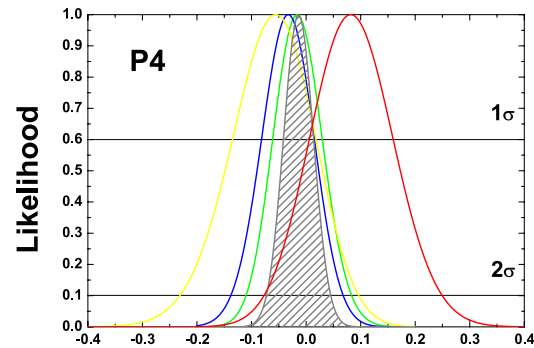

$\eta_{0}$

Figure 3. Likelihood curves for the parametrizations P1, P2, P3 and P4, each row corresponds to a different parametrization. By considering the left panels, the solid green, black, blue and yellow lines correspond to analyses by using separately $\mathrm{GC} 1+\mathrm{SNe}$ Ia data, $\mathrm{GC} 2+\mathrm{SNe}$ Ia data, $f_{\mathrm{SZE}}^{\mathrm{obs}} / f_{\mathrm{x}-\text { ray }}^{\text {obs }}$ data and $T_{\mathrm{CMB}}(z)$ in equation (51), respectively. On the other hand, by considering the right panels, the solid green, red, blue and yellow lines correspond to analyses by using GC1 + SNe Ia data, GC3 + SNe Ia data, $f_{\mathrm{SZE}} / f_{\mathrm{x}-\text { ray }}$ data, and $T_{\mathrm{CMB}}(z)$, respectively. In all panels, the dashed area are the results of the joint analysis by using these data set. 


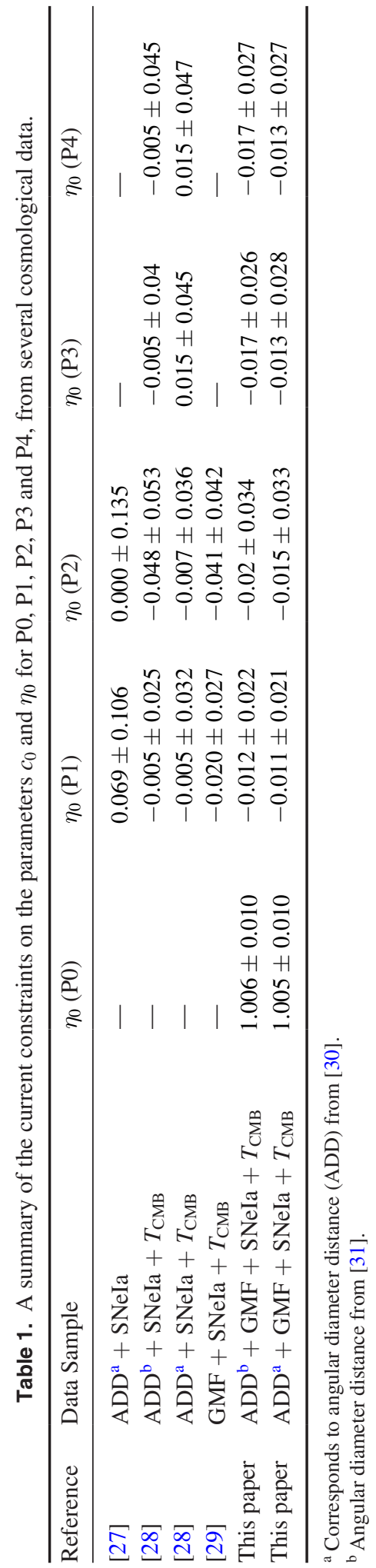




\section{Analises and results}

We evaluate our statistical analysis by defining the likelihood distribution function, $\mathcal{L} \propto e^{-\chi^{2} / 2}$, where

$$
\begin{aligned}
\chi^{2}= & \sum_{i=1}^{40} \frac{\left(\overline{\mu_{1}}\left(z_{i}\right)-\mu_{G C 1}\left(\eta, z_{i}\right)\right)^{2}}{\sigma_{\mathrm{obs}}^{2}}+\sum_{i=1}^{29} \frac{\left(\overline{\mu_{2}}\left(z_{i}\right)-\mu_{G C 2}\left(\eta, z_{i}\right)\right)^{2}}{\sigma_{\mathrm{obs}}^{2}}+\sum_{i=1}^{25} \frac{\left(\overline{\mu_{3}}\left(z_{i}\right)-\mu_{G C 3}\left(\eta, z_{i}\right)\right)^{2}}{\sigma_{\mathrm{obs}}^{2}} \\
& +\sum_{i=1}^{29} \frac{\left(\eta(z)^{2}-f_{\mathrm{SZE}}^{\mathrm{obs}} / f_{\mathrm{x}-\mathrm{ray}}^{\mathrm{obs}}\right)^{2}}{\sigma_{\mathrm{obs}}^{2}}+\sum_{i=1}^{38} \frac{\left[T\left(z_{i}\right)-T_{i, o b s}\right]^{2}}{\sigma_{T_{i}, o b s}^{2}}
\end{aligned}
$$

with GC1, GC2 and GC3 corresponding to samples from gas mass fraction [35], ADD [31] and ADD [30], respectively, and $\sigma_{\mathrm{obs}}^{2}=\sigma_{\bar{\mu}}^{2}+\sigma_{\mu G C}^{2}$ for each sample and $T(z)$ given by equation (49). The distance modulus $\overline{\mu_{1}}(i), \overline{\mu_{2}}(i)$ and $\overline{\mu_{3}}(i)$ correspond to weighted averages from the SNe Ia data for each i-galaxy cluster in samples present in [30, 31, 35] (see equation (50)). In our analysis, the normalization factor $N$ in equation (23) is taken as a nuisance parameter so that we marginalize over it. The EEP breaking is sought for allowing deviations from $\eta=1$ for parametrizations as (P1)-(P4), if $\eta_{0}=0$ the standard limit (with no interaction) for the electromagnetic sector is recovered.

The results for the parametrizations (P1), (P2), (P3) and (P4) are plotted in figure 3, each row depicting a different parametrization. The left panels show the results with the ADD data of Bonamente et al [31], while the right panels the ADD data of de Filippis et al [30]. By considering the left panels, the solid green, black, blue and yellow lines correspond to analyses by using separately GC1 + SNe Ia data, GC $2+$ SNe Ia data, $f_{\text {SZE }}^{\text {obs }} / f_{\mathrm{x}-\text { ray }}^{\text {obs }}$ data and $T_{\mathrm{CMB}}(z)$ in equation (51), respectively. The dashed area are the results of the joint analysis by using these data set. On the other hand, by considering the right panels, the solid green, red, blue and yellow lines correspond to analyses by using GC1 + SNe Ia data, GC3 + SNe Ia data, $f_{\mathrm{SZE}} / f_{\mathrm{x}-\text { ray }}$ data, and $T_{\mathrm{CMB}}(z)$, respectively. Again, the dashed area are the results of the joint analysis. As one may see from black and red lines, the results of the analyses by using $\mathrm{ADD}+\mathrm{SNe}$ Ia data do not depend strongly on the galaxy cluster sample used.

In table 1 , we put our $1 \sigma$ results from the joint analyses for the four parametrizations and several $\eta_{0}$ values already present in literature which consider correctly possible variations of $\alpha$ and $\eta$ in their analyses. For completeness we also added the case $\eta(z)=\eta_{0}\left(P_{0}\right)$, in this case the standard limit (with no interaction) in the electromagnetic sector is recovered for $\eta_{0}=0$. As one may see, our results are in full agreement with each other and with the previous ones regardless the galaxy cluster observations and $\eta(z)$ functions used. Moreover, our analysis presents most restrictive results and no significant break of EEP by means of the electromagnetic sector was verified.

\section{Conclusions}

The amount and quality of data gathered by cosmologists in the last decades allowed the establishment of a standard cosmological model, dubbed the flat $\Lambda$ CDM model. Along with it, these data also provide a myriad of opportunities to check the consistency of the cosmological framework and test for ideas beyond the standard model. One of the fundamental hypotheses of the cosmological framework is the validity of the Einstein equivalence principle (EEP). As it was recently shown in [7], a possible breakdown of the equivalence principle in the 
electromagnetic sector can demonstrate distinct signatures, for instance, deformations of the cosmic distance duality relation (CDDR) and a time variation of the fine structure constant.

In this paper, we have looked for possible deviations of the CDDR and a time-dependency of the fine structure constant as a test of the equivalence principle using four different observables at low and intermediate redshifts. The high complementarity of the samples due to their different degeneracies allowed us to improve constraints on the deviations of the CDDR between 20 and 40\%, depending on the parametrization adopted. The results point to a complete agreement with the validity of the EEP, which should be obeyed within a few percent regardless the considered parametrization. Future and ongoing surveys in different wavelengths will provide even more stringent tests to the EEP soon.

\section{Acknowledgments}

RFLH acknowledges financial support from CNPq (No. 303734/2014-0). SHP acknowledges financial support from CNPq-Conselho Nacional de Desenvolvimento Científico e Tecnológico, Brazilian research agency, for financial support, grants number 304297/2015-1 and 400924/2016-1. VCB is supported by São Paulo Research Foundation (FAPESP)/CAPES agreement under grant 2014/21098-1 and São Paulo Research Foundation under grant 2016/17271-5. CHGB is supported by CNPq under research project no 502029/2014-5.

\section{References}

[1] Hinterbichler K 2012 Rev. Mod. Phys. 84671

[2] Milgrom M 1983 Astrophys. J. 270365

[3] Sotiriou T P and Faraoni V 2010 Rev. Mod. Phys. 82451

[4] Randall L and Sundrum R 1999 Phys. Rev. Lett. 834690

[5] Falkowski A, Lalak Z and Pokorski S 2000 Phys. Lett. B 491172

[6] Bojowald M 2008 Living Rev. Relativ. 114

[7] Hees A, Minazzoli O and Larena J 2014 Phys. Rev. D 90124064

[8] Minazzoli O and Hees A 2014 Phys. Rev. D 90023017

[9] Hees A, Minazzoli O and Larena J 2015 Gen. Relativ. Gravit. 472

[10] Damour T and Polyakov A M 1994 Nucl. Phys. B 423532

[11] Damour T and Polyakov A M 1994 Gen. Relativ. Gravit. 261171

[12] Overduin J M and Wesson P S 1997 Phys. Rep. 283303

[13] Dine M, Fischler W and Srednicki M 1981 Phys. Lett. B 104199

[14] Kaplan D B 1985 Nucl. Phys. B 260215

[15] Bekenstein J D 1982 Phys. Rev. D 251527

[16] Sandvik H B, Barrow J D and Magueijo M 2002 Phys. Rev. Lett. 88031302

[17] Barrow J D and Lip S Z W 2012 Phys. Rev. D 85023514

[18] Barrow J D and Grahan A A H 2013 Phys.Rev. D 88103513

[19] Brax P et al 2004 Phys. Rev. D 70123518

[20] Brax P, van de Bruck C and Davies A C 2007 Phys. Rev. Lett. 99121103

[21] Ahlers M et al 2008 Phys. Rev. D 77015018

[22] Khoury J and Weltman A 2004 Phys. Rev. D 69044026

[23] Hargo T, Lobo F S N and Minazzoli O 2013 Phys. Rev. D 87047501

[24] Brans C and Dicke R H 1961 Phys. Rev. 124925

[25] Damour T and Dyson F 1996 Nucl. Phys. B 48037

[26] Murphy M T et al 2004 Lect. Notes Phys. 648131

[27] Holanda R F L and Barros K N N O 2016 Phys. Rev. D 94023524

[28] Holanda R F L and Pereira S H 2016 Phys. Rev. D 94104037

[29] Holanda R F L, Pereira S H and Santos-da-Costa S 2017 Phys. Rev. D 95084006

[30] De Filippis E, Sereno M, Bautz M W and Longo G 2005 ApJ 625108 
[31] Bonamente M et al 2006 Astrophys. J. 64725

[32] Suzuki N et al 2012 Astrophys. J. 85746

[33] Luzzi G et al 2009 Astrophys. J. 7051122

[34] Hurier G, Aghanim N, Douspis M and Pointecouteau E 2014 Astron. Astrophys. 561 A143

[35] Mantz A B et al 2014 Mon. Not. R. Astron. Soc. 4402077

[36] Minazzoli O and Hees A 2013 Phys. Rev. D 88041504

[37] Minazzoli O 2013 Phys. Rev. D 88027506

[38] Ellis G, Maartens R and MacCallum M 2012 Relativistic Cosmology (Cambridge: Cambridge University Press)

[39] Etherington I M H 1933 Phil. Mag. 15761

[40] Bassett B A and Kunz M 2004 Phys. Rev. D 69101305

[41] Holanda R F L, Lima J A S and Ribeiro M B 2011 Astron. Astrophys. 528 L14

[42] Li Z, Wu P and Yu W 2011 Astrophys. J. 729 L14

[43] Yang X et al 2013 Astrophys. J. Lett. 777 L24

[44] Holanda R F L and Busti V C 2014 Phys. Rev. D 89103517

[45] Holanda R F L, Busti V C and Alcaniz J S 2016 J. Cosmol. Astropart. Phys. JCAP02(2016)054

[46] Sunyaev R A and Zeldovich Ya B 1972 Comments Astrophys. Space Phys. 4173

[47] De Filippis E, Sereno M, Bautz M W and Longo G 2005 Astrophys. J. 625108

[48] Cavaliere A and Fusco-Fermiano R 1978 Astron. Astrophys. 66770

[49] Itoh N, Kohyama Y and Nozawa S 1998 Astrophys. J. 5027

[50] Galli S 2013 Phys. Rev. D 8712

[51] Uzan J P, Aghanim N and Mellier Y 2004 Phys. Rev. D 70083533

[52] Sarazin C L 1986 Astrophys. J. 1300

[53] Holanda R F L, Busti V C, Colaço L R, Alcaniz J S and Landau S J 2016 J. Cosmol. Astropart. Phys. JCAP08(2016)055

[54] Sasaki S 1996 PASJ 48 L119

[55] Grego L E A 2001 Astrophys. J. 5522

[56] La Roque S J et al 2006 Astrophys. J. 652917

[57] Holanda R F L, Landau S J, Alcaniz J S, Sanchez G I E and Busti V C 2016 J. Cosmol. Astropart. Phys. JCAP05(2016)047

[58] Holanda R F L, Gonçalves R S and Alcaniz J S 2012 J. Cosmol. Astropart. Phys. JCAP06(2012)022

[59] Allen S W, Schmidt R W and Fabian A C 2002 Mon. Not. R. Astron. Soc. 334 L1

[60] Allen S W, Schmidt R W, Ebeling H, Fabian A C and van Speybroeck L 2004 Mon. Not. R. Astron. Soc. 353457

[61] Ettori S et al 2009 Astron. Astrophys. 50161

[62] Gonçalves R S, Holanda R F L and Alcaniz J S 2012 Mon. Not. R. Astron. Soc. 420 L43

[63] Planelles S et al 2013 Mon. Not. R. Astron. Soc. 4311487

[64] Battaglia N, Bond J R, Pfrommer C and Sievers J L 2013 Astrophys. J. 777123

[65] Melchiorri F and Melchiorri B O 2005 Proc. of the Int. School of Physics Enrico Fermi vol $159 \mathrm{p}$ 225

[66] Guy J et al 2007 Astron. Astrophys. 46611

[67] Mather J C, Fixsen D J, Shafer R A, Mosier C and Wilkinson D T 1999 Astrophys. J. 512511 